\title{
Solution structure of human MBD1 CXXC1
}

\author{
Ross Thomson ${ }^{1} \cdot$ Brian O. Smith $^{1}$ (B)
}

Received: 12 August 2015 / Accepted: 2 September 2015/Published online: 9 September 2015

(C) The Author(s) 2015. This article is published with open access at Springerlink.com

\section{Biological content}

Methylation of a $\mathrm{CpG}$ dinucleotide at cytosine $\mathrm{C} 5(\mathrm{mCpG})$ is a major modification in vertebrate genomes associated with epigenetic gene silencing. $\mathrm{CpG}$ methylation recruits proteins which specifically recognise this motif and these methylated DNA binding proteins then recruit enzymes which chemically and physically alter chromatin, inducing transcriptional repression. Although most $\mathrm{CpG}$ motifs are methylated, short (500-2000 bp) CG-rich regions, known as $\mathrm{CpG}$ islands (CGIs), found within $\sim 60 \%$ of human gene promoters remain non-methylated (Bird 2002). How these CGIs contribute to epigentic regulation is an area of continuing research.

To investigate the possibility that proteins are targeted to these CGIs, Voo et al. (2000) used a ligand screen to identify proteins that bind non-methylated CpGs. They identified a protein, CFP1 (formerly CGBP) that contains a cysteine rich zinc finger-CXXC (ZF-CXXC) domain. Since this discovery, several more CXXC containing proteins have been identified as reviewed recently by Long et al. (2013). CXXC domains can be differentiated into three groups, type 1 which bind $\mathrm{CpG}$ and contain the KFGG motif, type 2 which do not bind $\mathrm{CpG}$ and lack the KFGG motif, and type 3 which lack the extended loop of types 1 and 2 but do bind to CpG (Fig. 3a). The conserved

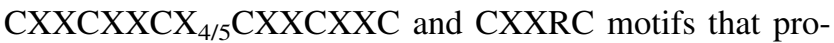
vide the amino acid residues that coordinate the two zinc

Brian O. Smith

Brian.Smith@glasgow.ac.uk

1 Institute of Molecular Cell and Systems Biology, College of Medical, Veterinary and Life Sciences, Joseph Black Building, University of Glasgow, Glasgow G12 8QQ, UK ions means that the main domain architecture, despite sequence variation between the three types, is similar in all CXXC domains.

CXXC domains are found in various chromatin binding proteins: CFP1 is a member of the SETDB1 complex responsible for H3K4 methylation; MLL protein is also a H3K4 methyltrasferase; KDM2A and KDM2B are H3K36 demethylase enzymes. DNMT1 and MBD1 are unique amongst CXXC domain containing proteins in that they can also bind hemi-methylated and methylated $\mathrm{CpG}$ respectively as well as non-methylated $\mathrm{CpG}$. As well as the methylated DNA binding domain, MBD1 also contains a transcriptional repressor domain (TRD) and depending on splice variant, 2 or 3 CXXC domains. The TRD is capable of transcriptional repression independently of the MBD domain, and MBD1 induces transcriptional repression by recruiting the histone $\mathrm{H} 3-\mathrm{K} 9$ methyl transferase enzyme SETDB1. After DNA replication, this histone methylation is heritably maintained due to MBD1 remaining at the replication fork through its interaction with chromatin assembly factor 1 . Once replication has occurred, DNMT1 activity fully methylates hemimethylated $\mathrm{CpG}$ motifs allowing MBD1 to rebind to the methyl-CpG (Sarraf and Stancheva 2004).

There are several published structures of type 1 (Allen et al. 2006; Cierpicki et al. 2010; Song et al. 2011; Xu et al. 2011) and type 3 (Xu et al. 2012) (unpublished data, PDB depositions 4BBQ, 4O64) CXXC domains with and without DNA bound that have revealed how the CXXC domain interacts with $\mathrm{CpG}$ DNA. The CXXC domains bind perpendicular to the DNA helical axis, with their DNA binding loops packed into the major groove. The type 1 DNA binding motif consists of three residues, in the case of hMLL, ${ }^{1185} \mathrm{KKQ}^{1187}$, which hydrogen bond to the CpG sequence. The main chain carbonyls of K1185 and K1186 
form hydrogen bonds to the $\mathrm{N} 4$ amines of the cytosine bases while the side chain amides of K1186 and Q1187 form hydrogen bonds to the guanines (Cierpicki et al. 2010). The first DNA binding residue is not strongly conserved amongst the type 1 CXXC domains, unlike the second and third which are either $\mathrm{KQ}$ or, in the case of CFP1 and MBD1 CXXC3, RQ. The type 3 domain DNA binding interface is similar and consists of XHQ but CXXC1 lacks these positively charged residues and CXXC2 only has one. The CXXC domains are unable to bind to methyl-CpG due to a steric clash between the methyl group and the protein backbone that prevents hydrogen bonding to the DNA bases.

Although MBD1 can contain up to 3 CXXC domains, only the third, type 1, CXXC domain is able to bind a $\mathrm{CpG}$ motif. MBD1 CXXC1 and 2 are type 2 domains and although similar to the type 1 in that they have an extended loop between the sixth and seventh cysteine they lack the conserved KFGG sequence and DNA binding motif. In this study we used NMR to determine the solution structure of the type $2 \mathrm{MBD} 1 \mathrm{CXXC1}$ domain in order to compare it to the published type $1 \mathrm{CXXC}$ structures. Using backbone dynamics we also investigated whether the lack of the KFGG motif in MBD1 CXXC1 affects the rigidity of the structure.

\section{Methods and results}

\section{Expression and purification of hMBD1 CXXC1}

MBD1 CXXC1 domain, residues 167-222, was amplified from the EST clone, accession number CF552871
(Geneservice Ltd ID 30529682) using the PCR primers: hMBD1 CXXC1 forward 5' GGGATCCGAGCAGAGAA TGTTTAAG $3^{\prime}$; hMBD1 CXXC1 reverse 5' CTCGAGT CAGCTCCTTTCCACAATC $3^{\prime}$. The plasmid pGEX-6p1 (GE healthcare) containing the CXXC domain from hMBD1 was over expressed in E. coli Tuner $^{\mathrm{TM}}$ DE3 cells (Novagen) as an N-terminal glutathione-S transferase (GST) fusion. Protein expression was induced $\left(\mathrm{OD}_{600}\right.$ 0.6-0.8) with $0.3 \mathrm{mM}$ IPTG at $13{ }^{\circ} \mathrm{C}$ overnight. Cells were harvested and resuspended in phosphate buffered saline solution (PBS) pH 7.3 (10 ml per 11 of culture). Bacteria were disrupted using BugBuster ${ }^{\mathrm{TM}}$ (Novagen) and DNA digested with Benzonase ${ }^{\circledR}$ nuclease (Novagen). The protein was purified by affinity chromatography using glutathione Sepharose 4 Fast Flow resin and the tag removed on-column by PreScission Protease. To remove EDTA from the PreScission protease it was first dialysed against

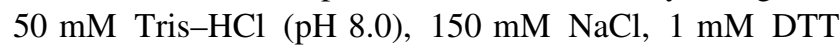
using a Thermo Scientific Slide-A-Lyzer Dialysis Cassette, 7000 molecular weight cut off (MWCO). Protein samples were buffer exchanged into $5 \mathrm{mM}$ deuterated imidazole, $250 \mathrm{mM} \mathrm{NaCl}, 1 \mathrm{mM}$ deuterated DTT and concentrated to a final volume of $570 \mu \mathrm{l}$ using a Vivaspin $205000 \mathrm{MWCO}$ centrifugal concentrator (Sartorius Stedim). For isotopically labelled protein, the bacteria were cultured using M9 minimal containing $1 \mathrm{~g} / \mathrm{l}{ }^{15} \mathrm{~N}$-ammonium chloride.

\section{NMR spectroscopy and NMR Resonance assignment}

NMR data were recorded on Bruker AVANCE $600 \mathrm{MHz}$ and $800 \mathrm{MHz}$ spectrometers equipped with $5 \mathrm{~mm}$ TCI cryoprobes using $1 \mathrm{mM}$ protein samples. $2 \mathrm{D}{ }^{1} \mathrm{H}$ TOCSY,

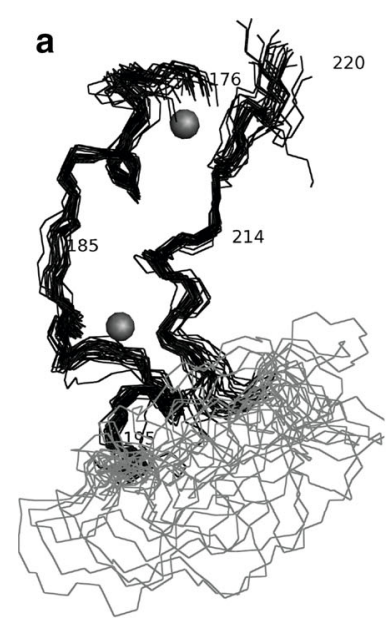

Fig. 1 The structure of hMBD1 CXXC1. a A stereo representation of the ensemble of MBD1 CXXC1 (residues 176-220) structures determined by NMR spectroscopy. The backbone trace is shown and selected residues labelled with the poorly defined loop (residues
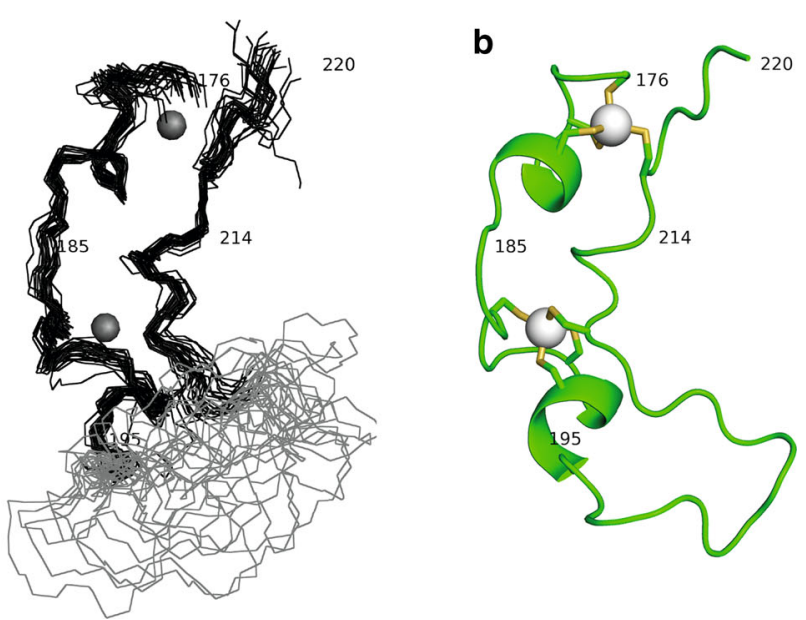

196-206) shown in grey. Zinc ions are shown as grey $1 \AA$ spheres. b Cartoon view of the solution structure of the representative structure from the ensemble with zinc atoms shown as grey spheres, each bonded to their coordinating cluster of cysteine residues 
$2 \mathrm{D}{ }^{1} \mathrm{H}$ NOESY and $2 \mathrm{D}{ }^{1} \mathrm{H}$ COSY spectra were recorded on unlabelled protein in samples containing $5 \% \mathrm{D}_{2} \mathrm{O}$ and $100 \% \mathrm{D}_{2} \mathrm{O} .2 \mathrm{D}{ }^{15} \mathrm{~N}$ HSQC, 3D ${ }^{15} \mathrm{~N}$ HSQC-TOCSY, 3D

${ }^{15} \mathrm{~N}$ HSQC-NOESY, HNHA and HNHB were recorded using ${ }^{15} \mathrm{~N}$ labelled protein. All NOESY experiments used for structure calculation were recorded with a mixing time of $100 \mathrm{~ms}$ and at a temperature of $293 \mathrm{~K}$. Data was processed with AZARA and analyzed with CCPN analysis software (Vranken et al. 2005). Maximum entropy reconstruction (Barna et al. 1987) was used to enhance the resolution of indirect dimensions in the 3D NOESY experiments.

Using the data obtained, $94.5 \%$ of the backbone and $86.5 \%$ of the side chain resonances where assigned for residues $167-222$. The aromatic side chain protons of the two phenylalanine residues $(171,207)$ were assigned by using the $2 \mathrm{D}{ }^{1} \mathrm{H}$ NOESY experiments recorded in $\mathrm{D}_{2} \mathrm{O}$ and in $\mathrm{H}_{2} \mathrm{O}$. The chemical shift assignments have been deposited with the BMRB under accession 25312.

${ }^{15} \mathrm{~N} T_{1}, T_{2}$ and heteronuclear-NOE relaxation data were recorded at $600.13 \mathrm{MHz}\left({ }^{1} \mathrm{H}\right)$ and $293 \mathrm{~K}$ and the data were analyzed according to the Lipari-Szabo model-free formalism using the program FAST-model free (Cole and Loria 2003). For $T_{1}$ measurements, data was recorded with relaxation delays of $0.1,1,2,4 \mathrm{~s}$ and for $T_{2}$ measurements, data was recoded with relaxation delays of 17 , $34,68,102,136,237 \mathrm{~ms}$. The recycle delay for both $T_{1}$ and $T_{2}$ experiments was $2.2 \mathrm{~s}$. Heteronuclear NOE values were determined from a pair of spectra recorded with and without proton saturation. The inter-scan relaxation delay for the heteronuclear NOE experiments was $6 \mathrm{~s}$ incorporating $3 \mathrm{~s}$ of proton saturation for the proton saturation experiment. The rotational correlation time $\left(\tau_{c}\right)$ for each residue was estimated using the $r 2 r l_{-} t m$ script (A. G. Palmer III, www.palmer.hs.columbia.edu/software. html), which calculates $\tau_{c}$ from the ratio of $\mathrm{R} 2 / \mathrm{R} 1$. Residues with NOE $<0.6$ or with an R2/R1 ratio more than one standard deviation from the mean were omitted. The molecular rotational time $\left(\tau_{\mathrm{m}}\right)$ of $5.67 \pm 0.141 \mathrm{~ns}$ for the entire molecule was then estimated by taking the mean $\tau_{\mathrm{c}}$ of the remaining residues.

\section{Structure calculation}

Structure calculations were carried out using ARIA 2.3 (Ambiguous Restraints for Iterative Assignment) (Rieping et al. 2007) over 8 iterations. 24 structures were calculated in iterations $1-5,50$ in iteration 6,100 in iteration 7 and 200 in iteration 8. 25 of the final 200 structures (Fig. 1a) were refined in a shell of water molecules with a full molecular dynamics force field incorporating electrostatics (Linge et al. 2003). Spin diffusion correction was included from iteration 3 using a distance cut off of $6 \AA$. In order to allow the use of spin diffusion correction during structure calculation, ARIA was used to derive distance restraints from the 3D ${ }^{15} \mathrm{~N}$ NOESY-HSQC and $2 \mathrm{D}{ }^{1} \mathrm{H}$ NOESY spectra. In addition to the 1327 NOE distance restraints, 38 ${ }^{3} \mathrm{~J}_{\mathrm{HN}-\mathrm{H} \alpha}$ (extracted from a 3D HNHA spectrum), $17{ }^{3} \mathrm{~J}_{\mathrm{HN}-\mathrm{H} \beta}$ restraints (derived from a 3D HNHB spectrum) and zinc co-ordination restraints for each of the cysteines (Cys 176, 179, 182 and 215 for zinc 1; Cys 188, 191, 194 and 210 for zinc 2) were also included and are summarised in Table 1.

Table 1 Structural statistics for the 20 lowest energy structures of hMBD1 CXXC1

\begin{tabular}{|c|c|}
\hline \multicolumn{2}{|l|}{ NMR Dist NMR distance restraints } \\
\hline Total NOE & 1327 \\
\hline Ambiguous & 593 \\
\hline Intra-residue & 204 \\
\hline \multicolumn{2}{|l|}{ Inter-residue } \\
\hline Sequential $(|\mathrm{i}-\mathrm{j}|=1)$ & 156 \\
\hline Short range $(1<|i-j| \leq 4)$ & 118 \\
\hline Long range $(|i-j| \geq 5)$ & 115 \\
\hline Unambiguous & 734 \\
\hline Intra-residue & 334 \\
\hline \multicolumn{2}{|l|}{ Inter-residue } \\
\hline Sequential $(|\mathrm{i}-\mathrm{j}|=1)$ & 211 \\
\hline Short range $(1<|i-j| \leq 4)$ & 102 \\
\hline Long range $(|i-j| \geq 5)$ & 87 \\
\hline \multicolumn{2}{|l|}{ Zinc co-ordination restraints } \\
\hline S-Zn bond length & $2.3 \AA$ \\
\hline $\mathrm{C} \alpha-\mathrm{C} \beta-\mathrm{S}$ bond angle & $114.3558^{\circ}$ \\
\hline $\mathrm{C} \beta-\mathrm{S}-\mathrm{Zn}$ bond angle & $109.5000^{\circ}$ \\
\hline $\mathrm{H} \beta-\mathrm{C} \beta-\mathrm{S}$ bond angle & $107.9185^{\circ}$ \\
\hline $\mathrm{S}-\mathrm{Zn}-\mathrm{S}$ bond angle & $109.5000^{\circ}$ \\
\hline \multicolumn{2}{|l|}{ Structural statistics (20 structures) } \\
\hline \multicolumn{2}{|l|}{ Constraint violations per structure } \\
\hline$>0.5 \AA( \pm \mathrm{SD})$ & $0.10( \pm 0.06)$ \\
\hline$>0.3 \AA( \pm \mathrm{SD})$ & $1.35( \pm 0.16)$ \\
\hline$>0.1 \AA( \pm \mathrm{SD})$ & $19.05( \pm 0.81)$ \\
\hline \multicolumn{2}{|l|}{ R.M.S. deviations from the ideal geometry } \\
\hline Bond length $(\AA)$ & $0.0038( \pm 0.000023)$ \\
\hline Bond Angle $\left({ }^{\circ}\right)$ & $0.600( \pm 0.05)$ \\
\hline Improper angles $\left({ }^{\circ}\right)$ & $1.476( \pm 0.04)$ \\
\hline Dihedrals $\left({ }^{\circ}\right)$ & $42.17( \pm 0.137)$ \\
\hline Ramachandran plot analysis & $\%$ of residues ${ }^{\mathrm{a}}$ \\
\hline Residues in most favoured regions & $61.3 \%(77.7 \%)$ \\
\hline Residues in additional allowed regions & $32.3 \%(21.0 \%)$ \\
\hline Residues in generously allowed regions & $3.3 \%(0.7 \%)$ \\
\hline Residues in disallowed regions & $3.1 \%(0.7 \%)$ \\
\hline
\end{tabular}

${ }^{a}$ Residues from well-defined regions of the structure, 175-196 and 208-216 
The assignment of the cysteine residues to the two zinc clusters was determined by initially calculating structures without zinc atoms or coordination restraints. In these calculations, the cysteine residues consistently clustered as indicated above. The zinc coordination restraints defined the bond lengths between the sulphur atoms of the cysteines and the zinc ion as well as the bond angles. The ensemble RMSD to the unbiased mean coordinates [determined using uwmn (Leo Caves, unpublished); uwmn implements an average distance matrix method to determine the unbiased mean structure which is then backprojected into cartesian space, and from which the ensemble RMSDs are calculated following superposition of the individual structures] for residues 175-196 and 208-217 of the water refined structures are $1.040 \AA$ (all heavy atoms) and $0.751 \AA$ (backbone heavy atoms) while PROCHECK-NMR (Laskowski et al. 1996) was used for validation. Greater than $90 \%$ of the residues fall within the most favorable and additionally allowed regions. However, the contribution of unfavorable $\varphi$ and $\psi$ angles in the unstructured N- (163-174) and C- (218-222) termini as well as the poorly defined loop region (197-207) results in higher than usual number of residues in the disallowed region. If the unstructured regions are removed from the Ramachandran analysis, the percentage of residues in the disallowed regions is reduced and the proportion of residues falling within the most favorable and additionally allowed regions increases to over $97 \%$. The NMR restraints and coordinates of the best 20 structures were deposited in the Protein Data Bank under accession code 4D4W.

\section{Discussion and conclusions}

\section{Overview of the solution structure of hMBD1 CXXC1}

The solution structure of MBD1 CXXC1 (residues 167-222) adopts a crescent shape incorporating two zinc ions (Fig. 1b). Four cysteines provide the ligands for the coordination of each zinc ion, three from each CXXCXXC motif. The main chain loops back $180^{\circ}$ after the second CXXCXXC motif providing the two further cysteines that complete the zinc coordination sites. The overall structure of the domain is governed by the presence of the two zinc clusters. Mutations of one of the zinc coordinating cysteines will destabilise the structure of the MLL CXXC domain (Allen et al. 2006. Cierpicki et al. 2010), MBD1 CXXC3 (Clouaire et al. 2010) and CGBP (Lee et al. 2001) suggesting the CXXCXXC motifs are absolutely required for maintaining the structure of the domain. The zinc clusters have similar structures to each other with a backbone RMSD of $0.86 \AA$ over the CXXCXXC sequence [calculated using Superpose (Maiti et al. 2004)] and form a small helical turn between the first and second cysteines followed by a single turn of alpha helix, that includes residues 180A-183Q (cluster 1) or (192S-196L) cluster 2.
Fig. 2 Backbone dynamics parameters of hMBD1 CXXC1. Extended Lipari-Szabo model free parameters derived from ${ }^{15} \mathrm{~N}$ relaxation parameters are presented for each residue where a motional model gave an acceptable fit. The overall correlation time was estimated as $5.6 \mathrm{~ns}$

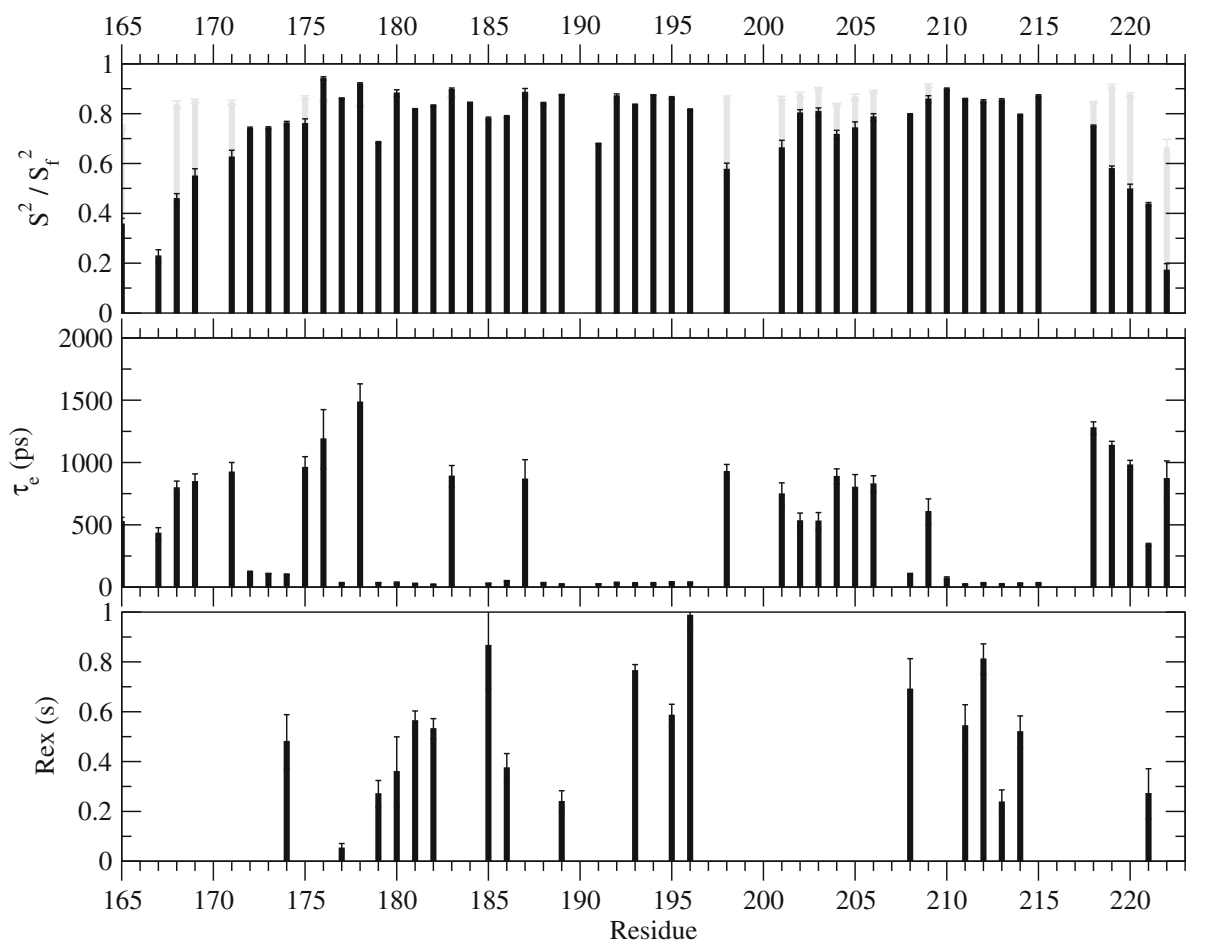


Residues 197-207 lack long range NOEs and thus form a flexible, poorly defined loop that is markedly different to the type 1 CXXC domain DNA binding loop region, while residues $167-174$ and $218-222$ form flexible $\mathrm{N}$ - and C-termini respectively.

\section{${ }^{15} \mathrm{~N}$ backbone dynamics}

Fast-model free was able to assign internal dynamic models to 47 out of 56 residues (Fig. 2), 1 represented by model $1\left(S^{2}\right), 8$ by model $2\left(S^{2}, \tau_{\mathrm{e}}\right), 18$ by model $4\left(S^{2}, \tau_{\mathrm{e}}\right.$, $\left.\mathrm{R}_{\mathrm{ex}}\right)$ and 20 by model $5\left(\mathrm{~S}^{2}, \tau_{\mathrm{e}}, \mathrm{S}_{\mathrm{f}}^{2}\right)$. Five of the eight residues were not fitted to any model (190A, 197Q, 207F, $216 \mathrm{~L}, 217 \mathrm{R}$ ), while two were not assessed because their amides could not be assigned $(170 \mathrm{M}, 200 \mathrm{H})$ and one is a proline residue (199P).

The results show the two termini and loop region are dynamic with low $\mathrm{S}^{2}$ values and internal correlation times on the pico- to nano-second time scale. The loop region is further characterised by requiring the $S_{2}^{\mathrm{f}}$ function to fit the internal motion to a model. Residues 175-196 and 208-217 have low coordinate RMSD over the structure ensemble and mean $S^{2}$ values 0.766 and 0.837 respectively, indicating only modest internal motion. The second cysteine in each cluster, C179 and C191, has a lower $S^{2}$ value, 0.684 and 0.679 respectively, indicating more internal motion compared to other residues in the well-structured region. Both cysteine clusters have rapid internal motion in the low ps time scale, while the region around cluster one also undergoes chemical exchange.

\section{Comparison to type 1 CXXC domains}

The solution structure of hMBD1 CXXC1 presented in this paper shows both similarities and differences to the known structures of type 1 CXXC domains (Allen et al. 2006; Cierpicki et al. 2010; Song et al. 2011; Xu et al. 2011) (Fig. 3b). The two zinc coordinating cysteine clusters of the CXXCXXC motifs form a common fold that is likely to be common to all other CXXC domains. However, hMBD1 CXXC1 lacks the KFGG motif that forms a small helix after the second CXXCXXC sequence present in type 1 non-methyl $\mathrm{CpG}$ binding CXXC domains. In hMBD1 CXXC1, the corresponding sequence, ${ }^{199} \mathrm{PHDV}^{202}$, has a significant effect on the structure of the loop region since P199 does not allow the hydrophobic side chain of H200 to pack up against $\beta$ protons of $\mathrm{C} 194$ to form a helix, resulting in an elongated loop (Fig. 3c). In xDNMT1 CXXC the KFGG sequence is preceded by a lysine rather than the proline in the MLL CXXC domain and the solution structure ensemble of XDNMT1 CXXC (Thomson and Smith, unpublished) reveals a less well defined loop region than hMLL CXXC, implying that the proline preceding the a

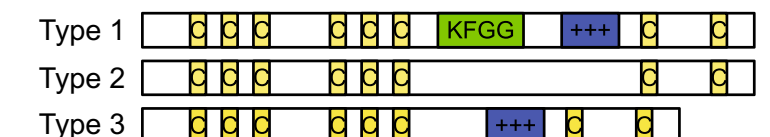

b

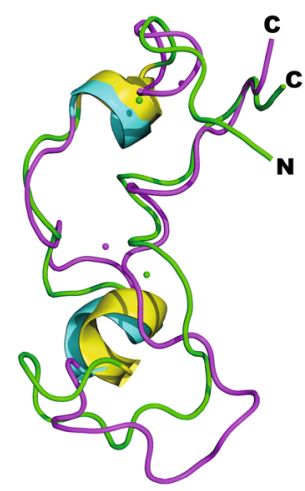

d
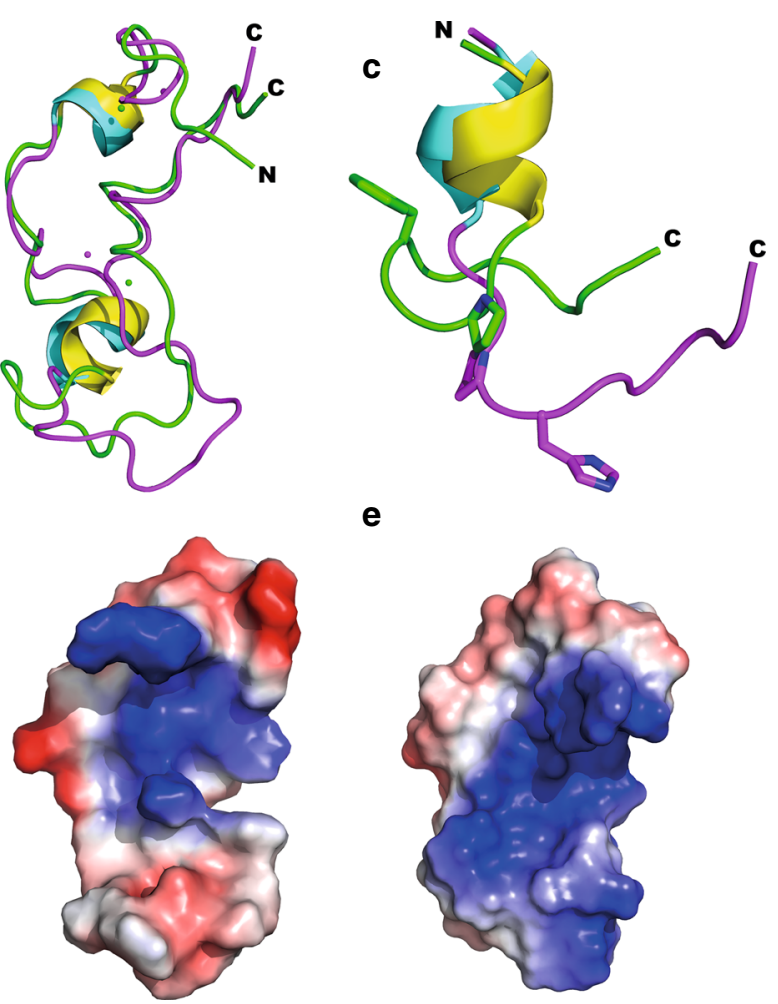

e

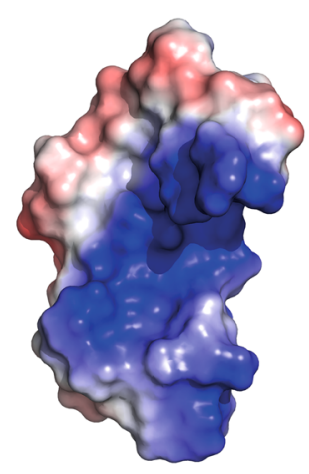

Fig. 3 a Schematic of CXXC domains showing the features of the three main types. The conserved Cys residues are indicated in yellow while the DNA-binding motifs are indicated in blue with plus signs. The KFGG sequence that rigidifies the loop of type 1 domains is highlighted. b Comparison of the structures of hMBD1 CXXC1 (magenta and cyan) and the MLL CXXC domain (green and yellow) and $\mathbf{c}$ close up of their loops showing how FXXX locks the loop to the second zinc cluster in MLL. d, e Comparison of the surface electrostatic potentials of hMBD1 CXXC1 and MLL CXXC, respectively, revealing a net negative charge on the loop region of hMBD1 CXXC1, contrasting with the positively charged DNA binding face for MLL CXXC

KFGG sequence may be required for loop rigidity. Mutation of hMLL CXXC residues K1178 or F1179 to alanine significantly reduced its ability to bind a $12 \mathrm{bp}$ single $\mathrm{CpG}$ DNA in a gel shift assay (Allen et al. 2006) indicating that the precise and ordered structure of the loop may be required for efficient DNA binding.

DNA binding domains are generally evolved to contain positively charged amino acids through which they contact the negatively charged DNA phosphate backbone (Jones et al. 2003) while the shape of the DNA binding face is often complementary to the DNA duplex surface (Tsuchiya et al. 2004). The surface charge plot of hMBD1 CXXC1 (Adaptive Poisson-Boltzmann Solver plugin, PyMol) reveals a positive charge surrounding the first CXXCXXC 
motif while the second CXXCXXC motif and loop region has an overall negative charge (Fig. 3d). This contrasts with the positively charged face of DNA binding loop of the hMLL CXXC domain (Fig. 3e). This is not surprising as hMBD1 CXXC1 lacks the conserved, positively charged residues found in type 1 domains required for binding to CG nucleotides.

The function of hMBD1 CXXC1 is currently unknown although it has been shown to bind residues 250-337 of Ring $1 b$ (a component of the Polycomb group (PcG) multiprotein PRC1 complex). Since hMBD1 CXXC3 does not interact with Ring1b (Sakamoto et al. 2007), the different loop region of hMBD1 CXXC1 may be involved in the protein interaction with Ring $1 b$.

Acknowledgments RT was supported by a BBSRC studentship funded under DTA BB/B511986/1.

Open Access This article is distributed under the terms of the Creative Commons Attribution 4.0 International License (http://crea tivecommons.org/licenses/by/4.0/), which permits unrestricted use, distribution, and reproduction in any medium, provided you give appropriate credit to the original author(s) and the source, provide a link to the Creative Commons license, and indicate if changes were made.

\section{References}

Allen M et al (2006) Solution structure of the nonmethyl-CpGbinding CXXC domain of the leukaemia-associated MLL histone methyltransferase. Embo J 25:4503-4512. doi:10.1038/ sj.emboj. 7601340

Barna JCJ, Laue ED, Mayger MR, Skilling J, Worrall SJP (1987) Exponential sampling, an alternative method for sampling in two-dimensional NMR experiments. J Magn Reson 73:69-77

Bird A (2002) DNA methylation patterns and epigenetic memory. Genes Dev 16:6-21. doi:10.1101/gad.947102

Cierpicki T et al (2010) Structure of the MLL CXXC domain-DNA complex and its functional role in MLL-AF9 leukemia. Nat Struct Mol Biol 17:U62-U82. doi:10.1038/nsmb.1714

Clouaire T, de Las Heras JI, Merusi C, Stancheva I (2010) Recruitment of MBD1 to target genes requires sequence-specific interaction of the MBD domain with methylated DNA. Nucleic Acids Res 38:4620-4634. doi:10.1093/nar/gkq228

Cole R, Loria J (2003) FAST-Modelfree: a program for rapid automated analysis of solution NMR spin-relaxation data. J Biomol NMR 26:203-213. doi:10.1023/A:1023808801134

Jones S, Shanahan HP, Berman HM, Thornton JM (2003) Using electrostatic potentials to predict DNA-binding sites on DNAbinding proteins. Nucleic Acids Res 31:7189-7198
Laskowski R, Rullmann J, MacArthur M, Kaptein R, Thornton J (1996) AQUA and PROCHECK-NMR: programs for checking the quality of protein structures solved by NMR. J Biomol NMR 8:477-486. doi:10.1007/BF00228148

Lee JH, Voo KS, Skalnik DG (2001) Identification and characterization of the DNA binding domain of CpG-binding protein. J Biol Chem 276:44669-44676. doi:10.1074/jbc.M107179200

Linge J, Williams M, Spronk C, Bonvin A, Nilges M (2003) Refinement of protein structures in explicit solvent. Protein Struct Funct Bioinform 50:496-506. doi:10.1002/prot.10299

Long H, Blackledge N, Klose R (2013) ZF-CxxC domain-containing proteins, $\mathrm{CpG}$ islands and the chromatin connection. Biochem Soc Trans 41:727-740. doi:10.1042/BST20130028

Maiti R, Van Domselaar GH, Zhang H, Wishart DS (2004) SuperPose: a simple server for sophisticated structural superposition. Nucleic Acids Res 32:W590-W594. doi:10.1093/nar/ gkh477

Rieping W, Habeck M, Bardiaux B, Bernard A, Malliavin TE, Nilges M (2007) ARIA2: automated NOE assignment and data integration in NMR structure calculation. Bioinformatics 23:381-382. doi:10.1093/bioinformatics/bt1589

Sakamoto Y, Watanabe S, Ichimura T, Kawasuji M, Koseki H, Baba H, Nakao M (2007) Overlapping roles of the methylated DNAbinding protein MBD1 and polycomb group proteins in transcriptional repression of HOXA genes and heterochromatin foci formation. J Biol Chem 282:16391-16400. doi:10.1074/jbc. M700011200

Sarraf SA, Stancheva I (2004) Methyl-CpG binding protein MBD1 couples histone $\mathrm{H} 3$ methylation at lysine 9 by SETDB1 to DNA replication and chromatin assembly. Mol Cell 15:595-605. doi:10.1016/j.molcel.2004.06.043

Song J, Rechkoblit O, Bestor T, Patel D (2011) Structure of DNMT1DNA complex reveals a role for autoinhibition in maintenance DNA methylation. Science 331:1036-1040. doi:10.1126/science. 1195380

Tsuchiya Y, Kinoshita K, Nakamura H (2004) Structure-based prediction of DNA-binding sites on proteins using the empirical preference of electrostatic potential and the shape of molecular surfaces. Proteins 55:885-894. doi:10.1002/prot.20111

Voo K, Carlone D, Jacobsen B, Flodin A, Skalnik D (2000) Cloning of a mammalian transcriptional activator that binds unmethylated $\mathrm{CpG}$ motifs and shares a CXXC domain with DNA methyltransferase, human trithorax, and methyl-CpG binding domain protein 1. Mol Cell Biol 20:2108-2121

Vranken W et al (2005) The CCPN data model for NMR spectroscopy: development of a software pipeline. Proteins Struct Funct Bioinform 59:687-696. doi:10.1002/prot.20449

Xu C, Bian C, Lam R, Dong A, Min J (2011) The structural basis for selective binding of non-methylated $\mathrm{CpG}$ islands by the CFP1 CXXC domain. Nat Commun. doi:10.1038/ncomms1237

$\mathrm{Xu} \mathrm{Y}$ et al (2012) Tet3 CXXC domain and dioxygenase activity cooperatively regulate key genes for Xenopus eye and neural development. Cell 151:1200-1213. doi:10.1016/j.cell.2012.11. 014 